\title{
Functional Design of English Online Examination System Based on ASP Technology

\author{
Yan Cong
}

Teaching and Research Institute of Foreign Languages, Bohai University, Jinzhou, 121013, China

cy9850@sina.com

Keywords: English teaching; online examination system; ASP.NET; ADO.NET technology; module structure; functional design

\begin{abstract}
With the continuous improvement of English at the college status, cultivating students' comprehensive ability as an important objective, so the test of the students' English proficiency is crucial, according to the shortcomings of ordinary paper exam, design English online examination system, both save manpower and resources, reduce the cost of the examination, but also improve efficiency and ensure fairness, to meet the needs of college English examination. In this paper, first describes the importance of English online examination; Secondly, to introduce this system based on $\mathrm{B} / \mathrm{S}$ development model, using ASP.NET as a programming environment, ADO.NET technology to access the database, in order to establish an efficient, interactive, secure server-side applications program. Then the modular structure of the system has been designed; finally describes the main function modules of the system.
\end{abstract}

\section{Introduction}

Along with the social requirements for students' English level increasing, college English is as an important part of college course, each institution have a large number of students going on public English learning, English exam is one of the methods to measure the students' English language proficiency, large number of students and the old way to modify the test paper, resulting in low efficiency, in order to fully ensure the fairness and impartiality of the examination, improve test efficiency, achieve autonomy in learning English, and realize the network of English examinations. a simple, practical, efficient and intelligent examination system, has become a necessary trend on college English learning development [1]. Can provide great convenience for learning, has the scientific, confidentiality, fairness, economy, practicality and maneuverability characteristics. The implementation of computer examination system on paperless, networking, automated, has far-reaching significance and practical value. Have a greater promoting role and application value for English learning curriculum construction and reform and the teachers themselves development.

\section{ASP.NET Technology}

ASP.NET is built on the basis of .NET platform architecture, on the back-end server provides robust enterprise-level Web application services programming framework for users, is a the server-side scripting technology that embed a web page script can executed by Internet server, it can be created dynamically on a Web server via HTTP requesting documents, its predecessor is ASP (Active Server Pages) technology. ASP.NET system structure is shown in Fig. 1.

ASP.NET technology represents the trend of Web programming technology, developed by Microsoft is a server-side scripting environment. By ASP.NET technology can combine HTML pages, ASP.NET commands, COM components, and stored procedures to create dynamic, interactive and efficient Web server applications program. Which has the following characteristics: independent of the browser, ASP script on the server side to explain the implementation, the client simply use regular HTML code interpretable browser, can browse the ASP.NET designed home; Object-oriented, in ASP scripts can be easily reference system components and ASP's built-in components, but also to extend the functionality through customized ActiveX Server Component; conveniently access to the database, ASP belongs to the server-side technologies in ActiveX 
technology, which is a executed script in server-side [2]. ASP.NET the biggest advantage is the use of ADO.NET technology to access the database, ADO.NET is a set of optimized and access to the database dedicated object set, for ASP provides a complete site database access scheme.

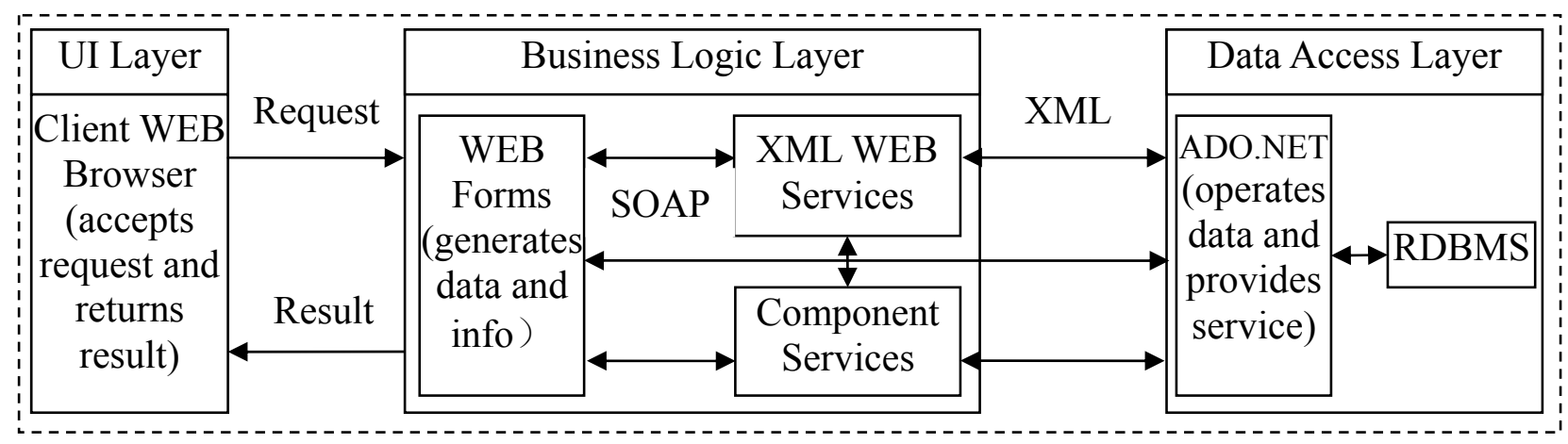

Fig. 1. ASP.NET system block diagram

\section{ADO.NET Technology}

ADO.NET (ActiveX Data Object.NET, Data Access Service) is a COM component library, It is a set of classes that .NET platform dedicated to access back-end database and data operations. It is an improved version of ADO, its functions is more powerful than ADO. ADO.NET does not depend on OLEDB to provide program, but the use of .NET hosting provide program, can disconnect Connection while retaining the current data sets are available, ADO.NET also introduces object-oriented structure of the past that are not, so database applications programming more structured. In addition, ADO.NET also uses standard XML as a data interchange format, so that different systems on the network can operate with each other. The structure of ADO.NET is shown in Fig. 2.

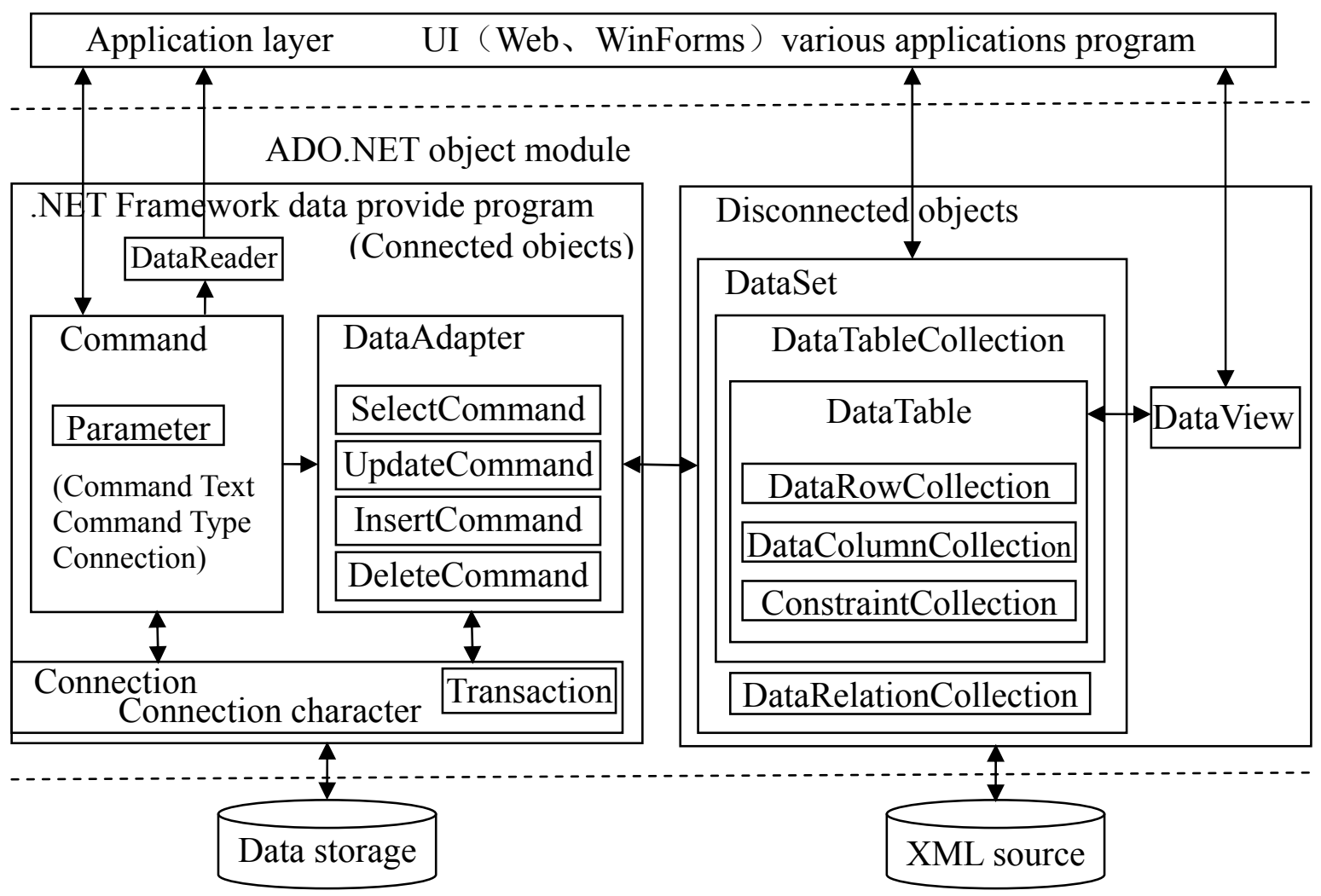

Fig. 2. ADO.NET structure

ADO.NET to access the database usually has two ways: one is through the Command object and 
DataReader object; another is through the DataAdapter object and the Dataset object [3]. As shown in Fig. 3. ADO.NET general procedures for accessing the database are: import the appropriate namespace. Establish a connection to the database, can use the SqlConnection object or OleDbConnection object. Execute SQL commands to the database in order to implement the query, insert, update and delete operations. For example, use the Command object or DatAdapter object to perform the SQL select, insert, update, or delete commands. Recordset retrieved from the database to fill the Dataset. Close the connection to the database. Do the required operating on the Dataset. If necessary, modify the data in the database, the result will be written back to the database.

ADO.NET has many advantages, making the data during the operation process becomes easy: interoperability, with components developed by different tools can communicate through the data storage. Performance, the data stored in ADO.NET is transmitted with XML format, does not need to data type conversion process, improve the efficiency of access; while in the early ADO, when the record set by means of COM components to transfer data, record set data must be converted to COM data types. Programmability, can use a variety of programming languages, is strongly typed programming environment [4].

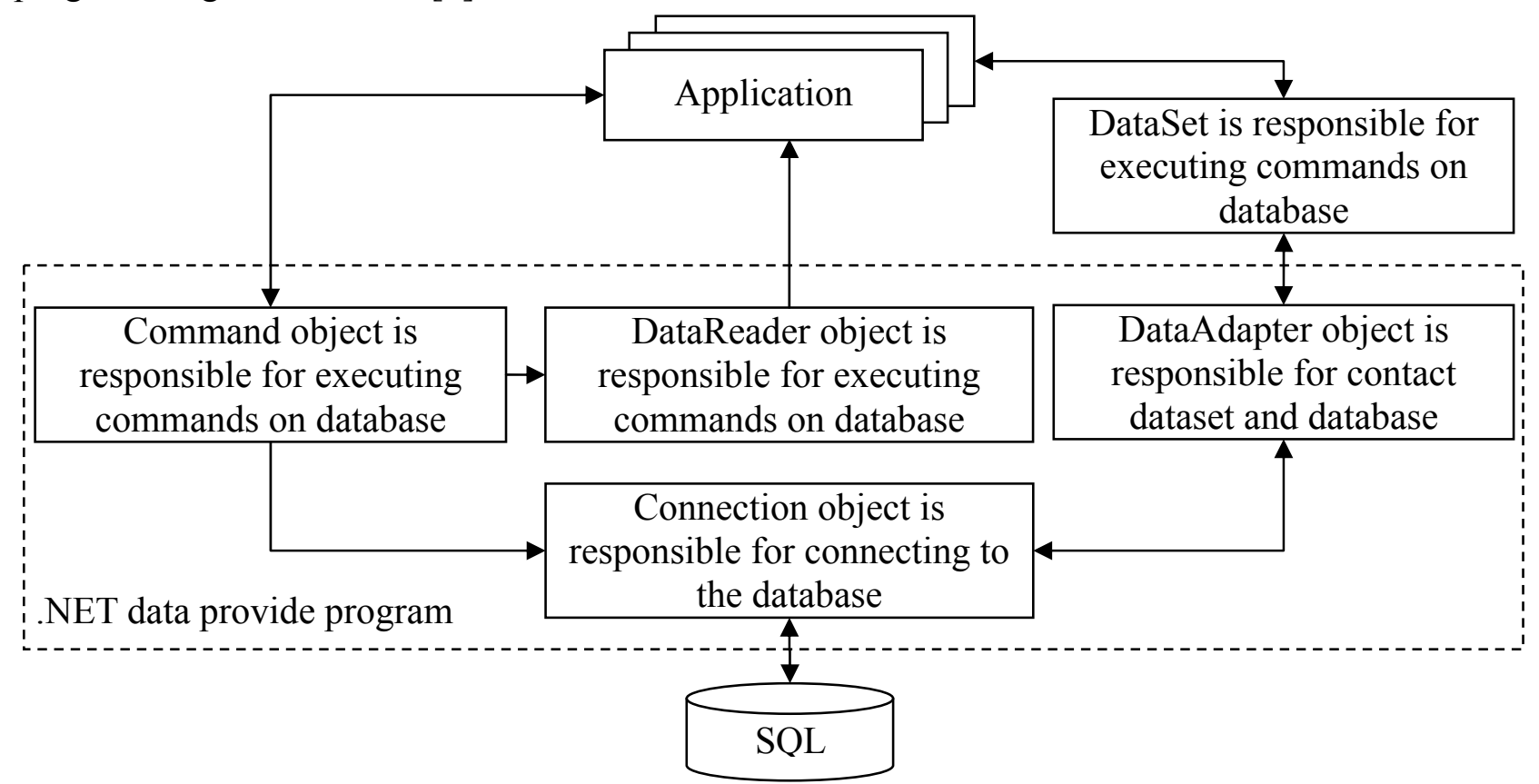

Fig. 3. The steps of ADO.NET to access the database

\section{Module Structure Chart Design}

Module structure chart (SC), also known as program structure chart. It is an important means that using structured design method to software summary design. The situation is different with data flow chart reflects data flow. Structure chart reflects the situation of control flow. In the structure chart, can be seen is contact and communication between the module and the module. The design process is that a complex system is decomposed into relatively simple multiple functions process. This decomposition into multiple functional modules relatively single method called modularity. Modularity is an important design thinking, this idea to a complex system is decomposed into a number of smaller-scale, features a more simple and easier to create and modify parts, on the one hand, each module has a relative independence, can respectively design and implement, on the other hand, the relationship between the modules (such as the exchange of information, the relationship between the call), then will be described through some ways. Each module within the constraints of these relationships together constitute a unified whole, complete the each function of the system.

Structured design methods is suitable for the overall design of software system, is developed based on modular, top-down program design, structure and so on, it is starting from the structure of the whole program, a design method of highlighting the program modules; The determined 
structures adopts orient throughout the system's data flow approach, while the quality of the software architecture provides a guiding principle. The basic idea is that the design of the system into the structure by relatively independent, single-function module composed. When using a structured design method for system design, generally use two steps: The first step is establishing the initial structure chart. The second step is improving the initial structure chart [5]. The key is to properly divide the module, using heuristic methods to deal with the linkages between modules, gradually achieve better design effect. Functional module structure of the system is shown in Fig. 4.

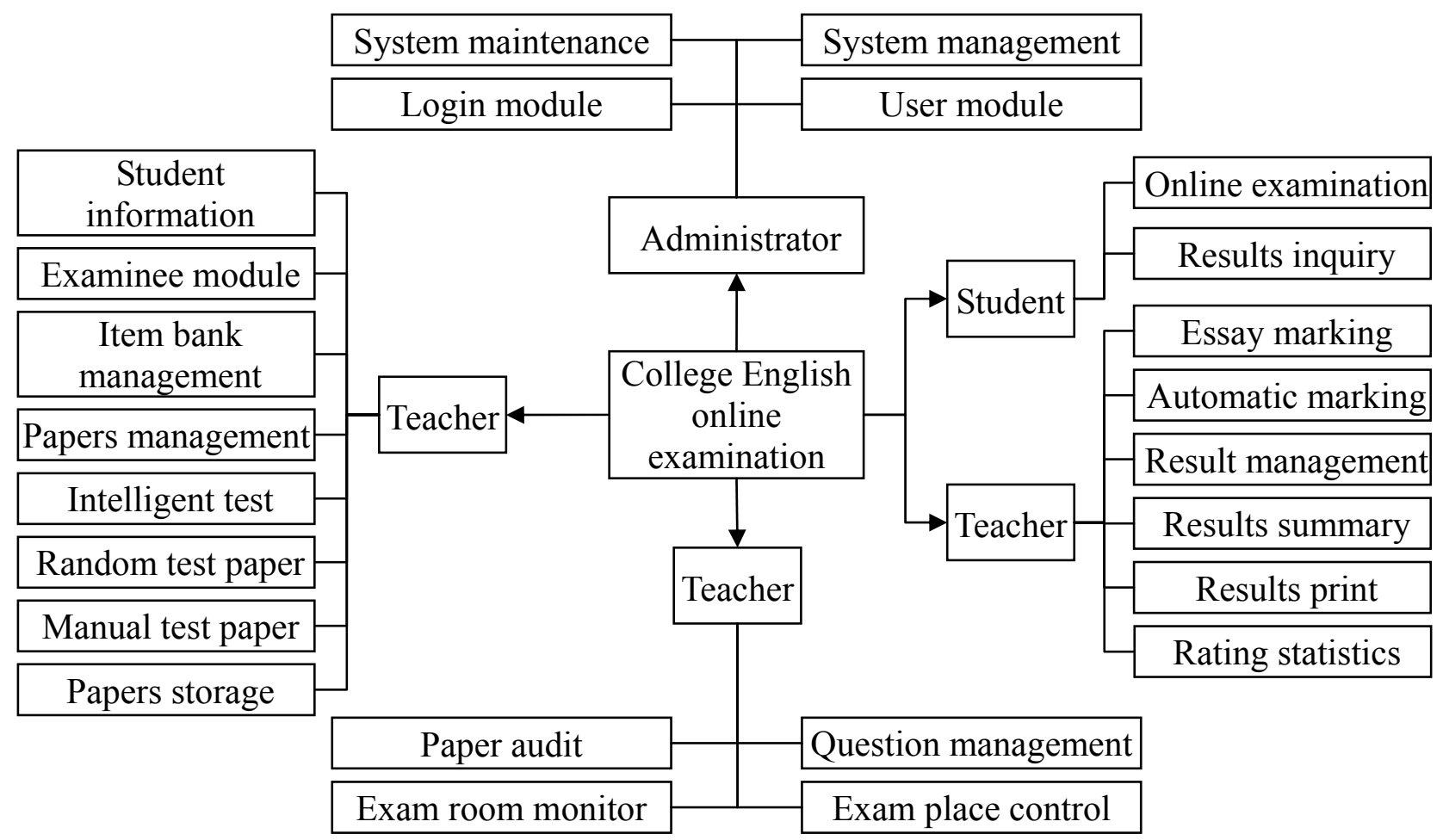

Fig. 4. Functional module structure chart of the system

\section{Functional Module Description}

The main function modules in Fig. 4 are described as follows:

Student information management module, the student information is stored according to department division, maintenance the departments' information by the administrator to perform. The system can query, modify, add, delete operations for department' information, such as department canceled English curriculum learning, directly delete the departments' information [6]. Another important function is to achieve and access to college educational management system, which can achieve a bulk import of student information, and student achievement to bulk import educational management system.

Test paper combining module, the system can be set up the random test paper combining in accordance with test paper subjects, knowledge, difficulty, question type, and total number of question projects, such as the title, but also can achieve low repetition of questions by using the improved genetic algorithm (use rate of same test papers in the past two years), with moderate difficulty and reasonable, to complete intelligent combining of the test paper [7]. Teacher can also manually test paper combining, or on the results of automatic test paper combining manually modified.

Online examination module, it is mainly responsible for the whole process that is the students from start to finish the exam, including student login, online exam, exam time, handed test paper and save functions. Intelligent test paper combining module contained in this module, at the beginning of the exam, will call test paper combining module, the module will be randomly generated a reasonable and scientific test paper, detailed information and the examinee obtained 
randomly test answers are saved to the corresponding data table [8].

Examination room monitoring module, each examinee has a corresponding section, namely the examinee status bar, a total of seven state, respectively is: not logged, are logged in, logged on, taking an exam, abnormal exit, reexamination, the end of the examination, the invigilator may very intuitive derive a examinee status information, in addition, after the server side start the exam instruction, in the exam monitoring window, the invigilator can also view each examinee's answer situation [9].

Performance management module, generating the achievement function mainly to integrate exam results of all examinee, and in accordance with the score from high to low or from low to high to order. This function is executed by the administrator or teacher. Pre-conditions for this function is performed by the system can all topics of automatically score are automatic scoring, subjective questions section has been completed manually scoring. Otherwise generate scores function cannot be executed. The generated results can be in tabular form preview, can also choose to print the ranking table. Through the query generated results table, can understand examination scores of a specified student number or name. Students can query their performance, if necessary, can also review their test papers (there is a time limit, such as within a week after the results announced).

\section{Conclusion}

With the development of society and the reform of English education, English examination among higher education has become a compelling important part. Because of the position of English class and scale of examination, English online exam certainly is a convenient way of examination. The system realizes the examinee can smoothly conduct online exams. Teachers for real time monitoring of the entire exam, administrators can easily maintain and manage the system. Teachers can be updated at any time for questions, knowledge point to modify emphasis and difficulty, and can check student achievement and comparison. Reach the demand goal of college English online examination, saving manpower and resources, reducing the cost of the exam and meet the needs of college English examination.

\section{References}

[1] T. Qi, "Design and Implementation of University Online Examination Management System," Xiamen University, 2014,5.

[2] J. G. Wang, "Design and Implementation of English Examination System Based on B/S Structure," Computer Engineering and Design, vol. 25, no. 6, pp.1011-1014, 2004.

[3] Y. D. Zhang, "Design and implementation of ASP.NET Adaptive Exam System," Nanjing University of Science and Technology, 2010.

[4] P. Hou, "The Design and Implementation of Test System of Speaking English," Tianjin Normal University, 2008.

[5] BaiduBaike. Module Design. http://baike.baidu.com/view/1679113.htm, 2014-9-25.

[6] B. B. Ning, "Implementation of Computer Examination System of University English," Northeast Normal University, 2013,5.

[7] N. Zhong, "Research of University English Online Homework and Online Examination System," China Educational Technology and Equipment, vol. 27, no. 6, pp.52-56, 2013.

[8] K. Lu, "Design and Implementation of Online Examination System in Colleges," Science and Technology Information, vol. 26, no.2 6, pp.233-234, 2010.

[9] Y. J. Zhang, "Design and Implementation of Examination System Without Paper Titles in English Based on C/S," University of Electronic Science and technology, 2010. 